CASE REPORT

\author{
M. Ribeiro \\ P. Howard \\ R. Willinsky \\ K. ter Brugge \\ R. Agid \\ L. Thines \\ L. da Costa
}

\section{Intrasellar Rupture of a Paraclinoid Aneurysm with Subarachnoid Hemorrhage: Usefulness of MR Imaging in Diagnosis}

\begin{abstract}
SUMmARY: Characterization of paraclinoid aneurysms may be difficult because of the complexity of anatomic structures involved, and differentiation between intradural and extradural lesions is crucial. We report a case of a patient with a unique presentation of a paraclinoid aneurysm with intrasellar hemorrhage in which the presence of intrasellar blood and the relationship of the paraclinoid aneurysmal neck and sac to the dural rings were elegantly demonstrated on MR imaging and were critical in choosing the target lesion for treatment.
\end{abstract}

$\mathbf{T}$ he anatomy of the paraclinoid region is complex, and sometimes it is hard to properly characterize aneurysms arising in that region. Differentiation between an intradural and extradural location is of the utmost importance because it may determine patient prognosis and management strategy. Intracavernous internal carotid artery (ICA) aneurysms usually have a benign natural history, and treatment is reserved for patients with unbearable pain or progressive neurologic deficits. An intradural aneurysm carries a risk for subarachnoid hemorrhage and may require treatment. We report an unusual case of multiple intracranial aneurysms including a ruptured paraclinoid carotid aneurysm presenting with intrasellar bleeding followed by subarachnoid extension.

\section{Case Report}

A 45-year-old man presented with good clinical grade subarachnoid hemorrhage. CT revealed blood in the basal cisterns and Sylvian fissures with symmetric distribution (Fig $1 A, B$ ). A digital subtraction angiography (Fig $1 C, D$ ) and a CT angiogram (CTA) demonstrated 2 aneurysms, a larger one arising from the right paraclinoid carotid and a smaller one at the right middle cerebral artery (MCA) bifurcation. The neck of the paraclinoid aneurysm could be seen at the level of the optic strut, with the sac pointing posteriorly and inferomedially, in close relationship with the sella turcica. (Fig 2). MR imaging was performed with the hope of being able to clarify if the paraclinoid aneurysm was intradural or extradural; results showed the aneurysmal neck to be intradural and its dome below the dural ring, extradural. In addition, an enlarged sella turcica was noted, with inferior displacement of the pituitary gland and a large amount of blood filling the sella and extending into the subarachnoid space (Fig 3). We concluded that the paraclinoid aneurysm had ruptured into the sella with subarachnoid extension. The paraclinoid aneurysm was successfully coiled.

Received November 20, 2007; accepted December 20.

From the Department of Neuroradiology (M.R.), Hospital São Marcos, Braga, Portugal; and Department of Medical Imaging (P.H., R.W., K.t.B., R.A., L.d.C.) and Division of Neurosurgery (L.T.), Department of Surgery, Toronto Western Hospital, University of Toronto, Toronto, Ontario, Canada.

Please address correspondence to Manuel Ribeiro, MD, Hospital São Marcos, Largo Engenheiro Carlos Amarante, Apartado 2242, Braga, Portugal, 4701-965; e-mail: manuelqr@portugalmail.pt

DOI 10.3174/ajnr.A1022

\section{Discussion}

The establishment of either an intradural or extradural location of paraclinoid aneurysms has critical implications for treatment and prognosis. Extradural aneurysms have a good prognosis and very low risks for a subarachnoid hemorrhage and major neurologic complications. ${ }^{1}$ Treatment is reserved for symptomatic lesions or for those lesions with extension into the subarachnoid space. ${ }^{2}$ Intradural aneurysms carry a risk for subarachnoid hemorrhage and may require treatment. Most paraclinoid aneurysms can be localized by their direction of projection on angiograms. ${ }^{3}$ However, the complex anatomy of the juxtadural ring area and individual variability in this region can sometimes make it difficult to define the relationship of the aneurysm to the dural ring, which differentiates extradural (below the dural ring) and intradural (above the dural ring) lesions.

Different techniques with several anatomic references have been used to differentiate intradural from extradural aneurysms. The simplest method uses the ophthalmic artery as the landmark for the transition of the extradural and intradural location. The major drawback of this method is that the origin of the ophthalmic artery is extradural in $10 \%$ of cases, ${ }^{4}$ leading to the erroneous assumption that an aneurysm is intradural when it is intracavernous. In our patient, the aneurysm arises distal to the ophthalmic artery, but its inferior direction places it below the plane of this artery (Fig 1D).

A reliable landmark for identification of the proximal dural ring, which defines the roof of cavernous sinus, is the optic strut. ${ }^{4-6}$ Lesions above this level are considered intradural, and below, intracavernous, extradural. In our case, the neck of the aneurysm is located at the exact level of the optic strut, and its fundus projects downward and medially, into the sella turcica. The aneurysm could be classified as a carotid cave aneurysm, arising between the 2 dural rings and growing into the cavernous sinus. The carotid cave is a pouch located in the posteromedial side of the distal dural ring, ${ }^{7}$ above the proximal ring, which may or may not have a communication with the subarachnoid space.

The diffuse subarachnoid hemorrhage demonstrated on CT could have been caused by either lesion, so it was important to determine if the proximal aneurysm was intradural or extradural. If the paraclinoid aneurysm was extradural, our treatment choice for the MCA aneurysm (Fig 1C) would have 

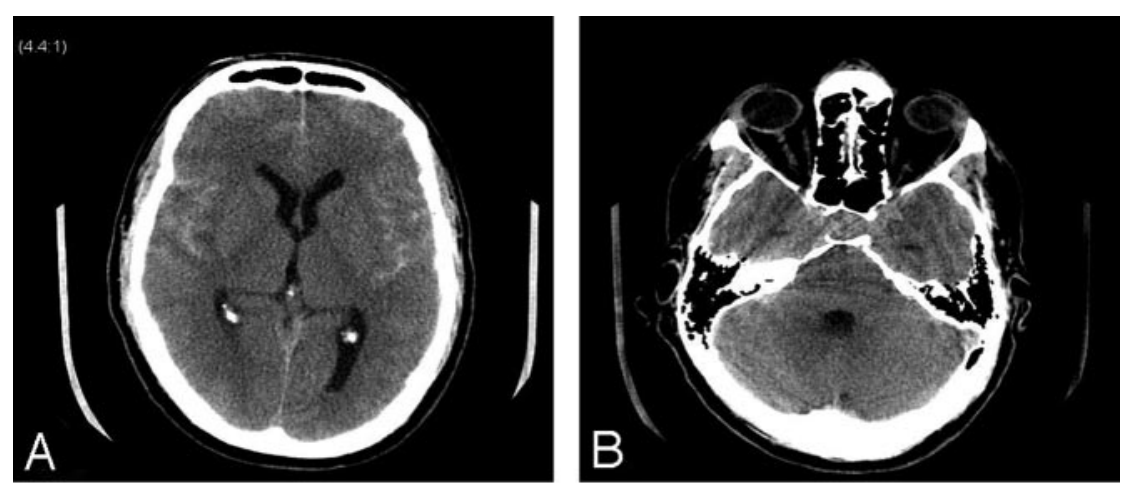

Fig 1. $A$, Axial unenhanced CT shows diffuse symmetric subarachnoid hemorrhage. $B$, Questionable hyperattenuation at the level of the sella turcica is difficult to interpret given adjacent streak artifact. C, Frontal view of right interna carotid angiogram shows MCA bifurcation and paraclinoid aneurysms. $D, 3 D$ right ICA angiogram in lateral view reveals the paraclinoid aneurysm arising distal to the ophthalmic artery but pointing inferiorly below the plane of the ophthalmic artery.
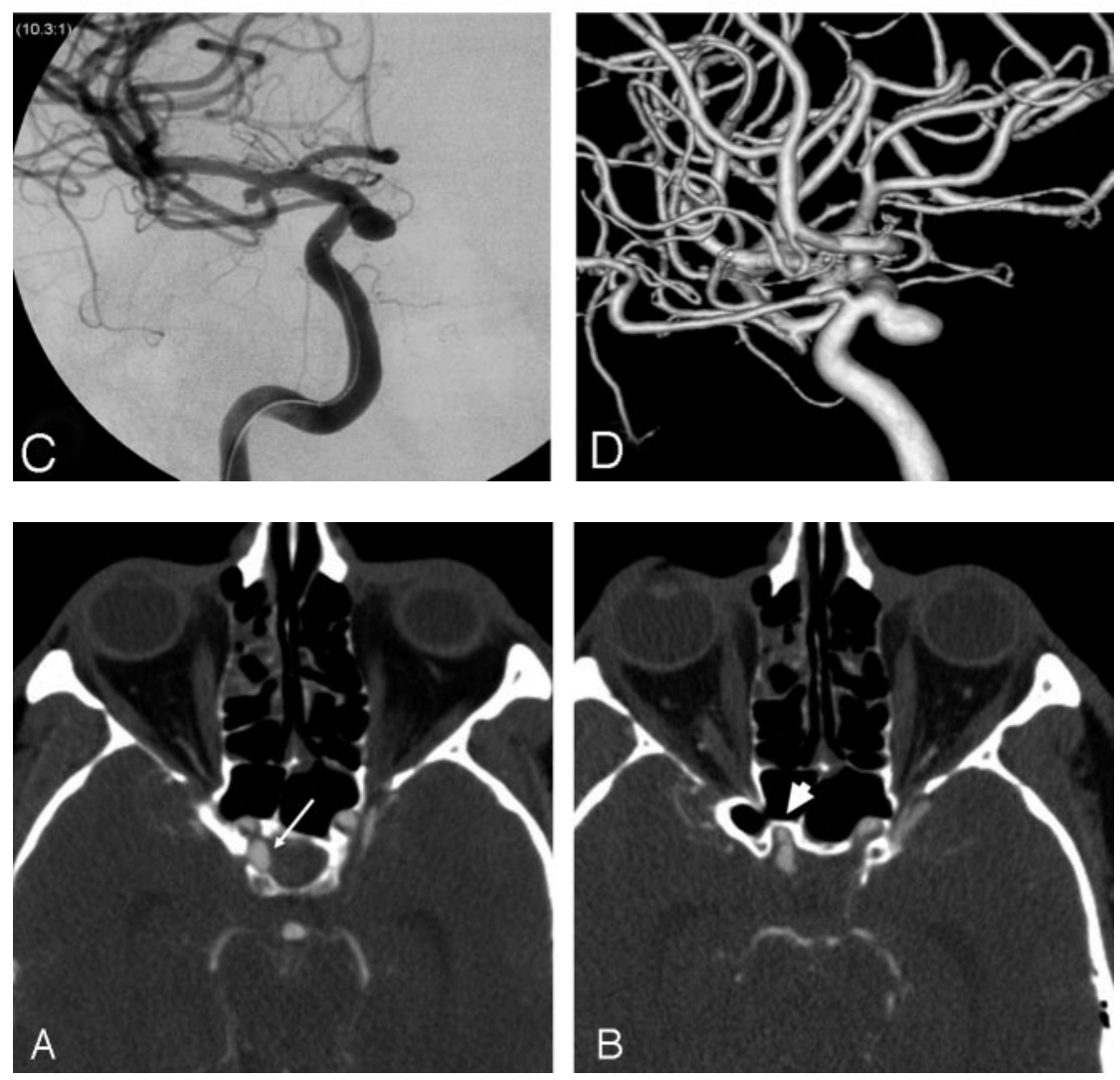

Fig 2. A, Axial CT angiography shows the sac of the paraclinoid aneurysm (arrow) adjacent to the sella, without intervening septum. $B$, At the level of the superior margin of the optic strut (arrowhead), the aneurysmal neck and body extend posteriorly from the ICA

been clipping (small size, relatively wide neck), but if it was intradural, our first choice of treatment would be coiling. CT and CTA alone were insufficient to define the location of the paraclinoid aneurysm and did not reveal the large intrasellar hemorrhage. In retrospect, after MR imaging revealed the intrasellar hemorrhage, intrasellar blood could be suspected on the plain CT head (Fig 1B).

MR imaging at 3T was used to visualize the dural ring $^{8}$ and showed that the neck of the aneurysm was intradural and its dome, clearly extradural. More importantly, it revealed a large amount of blood inside an enlarged sella turcica, confirming that the paraclinoid aneurysm had bled. The large amount of intrasellar blood, the relatively small volume of blood in the subarachnoid space, and the empty sella syndrome lead to the hypothesis that the aneurysm had bled primarily into the sella and secondarily through an incompetent diaphragma sellae into the subarachnoid space. Absence of a bony septum in the posteromedial segment of the carotid cave ${ }^{9}$ allows direct contact of the pulsatile aneurysmal dome with the dura, which eventually could cause its progressive erosion and the intrasellar hemorrhage. Advanced MR imaging techniques have improved our ability to identify the ruptured lesion in cases of multiple intracranial aneurysms, where MR imaging may identify a parenchymal clot adjacent to an aneurysm and recently has been used to determine an intradural or extradural location in unruptured paraclinoid aneurysms.

\section{Conclusion}

We report the case of a patient with a unique presentation of a paraclinoid aneurysm with intrasellar hemorrhage. Lesions located in the paraclinoid carotid can be difficult to clearly define as intradural or extradural. In this case, localization of the paraclinoid lesion (intradural vs extradural) and identification of the aneurysm responsible for the subarachnoid hemorrhage were critical in choosing a treatment strategy. The presence of intrasellar blood and the relationship of the paraclinoid aneurysm to the dura elegantly demonstrated on MR 

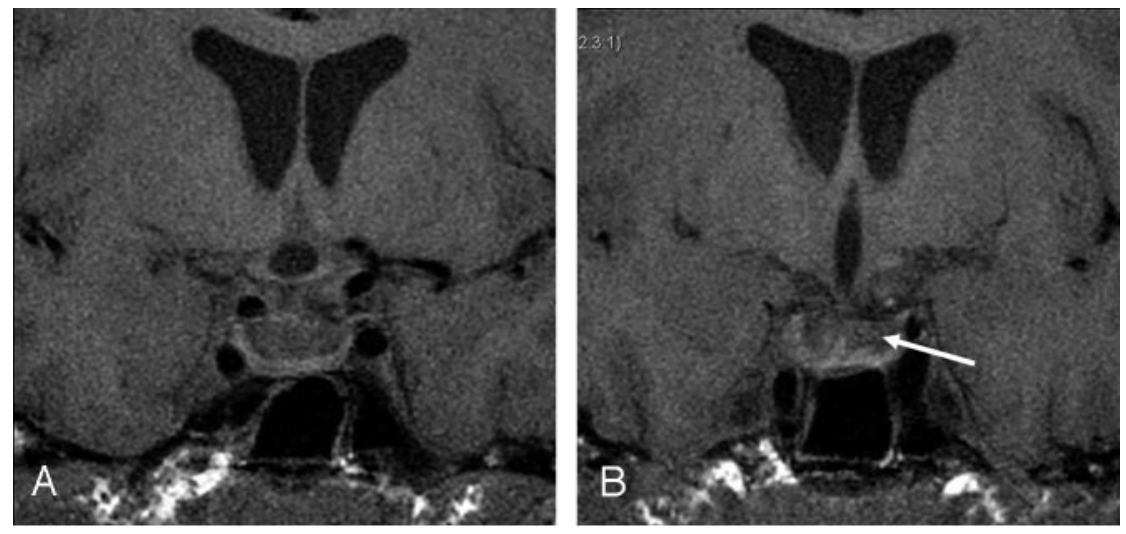

Fig 3. Coronal T1- and T2-weighted $3 \mathrm{~T} M \mathrm{MR}$ imaging through anterior $(A, C)$ and posterior $(B, D)$ sella. $C$, Junction of aneurysmal neck and body $(*)$, which projects partly superior to dural ring (interface with CSA and partly inferior to the dural ring (interface with blood within sella). Note the large sella turcica filled with blood (arrows) showing homogeneous hypointensity on T2-weighted images and isointensity on T1-weighted images, consistent with intracellular deoxyhemoglobin. Note that the blood in the suprasellar cisterns has different signal intensity. $D$, The infundibulum can be traced to the compressed pituitary.
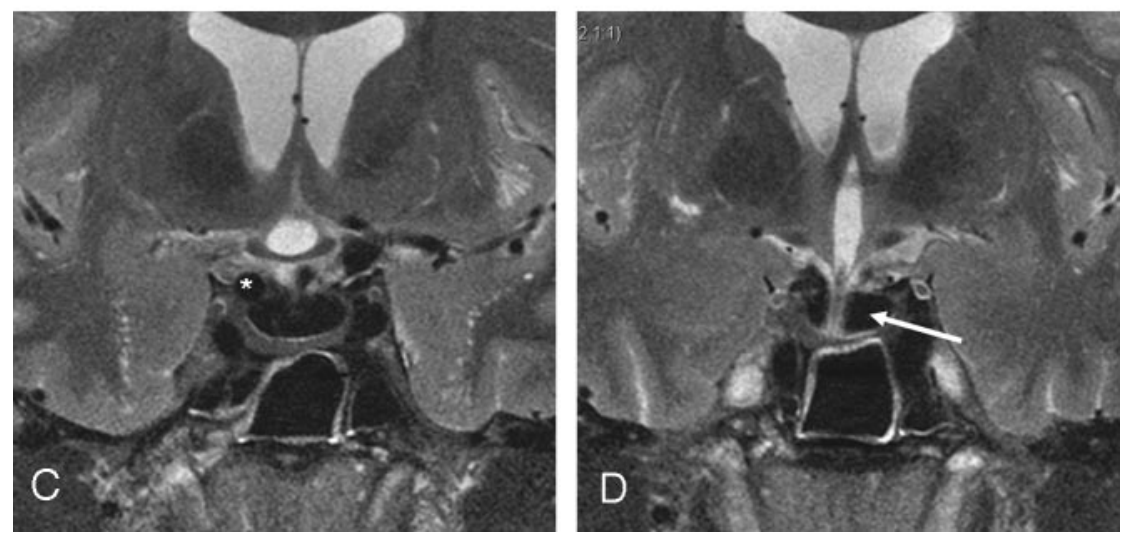

imaging were critical in choosing the target lesion for treatment.

\section{References}

1. Kupersmith MJ, Stiebel-Kalish H, Huna-Baron R, et al. Cavernous carotid aneurysms rarely cause subarachnoid hemorrhage or major neurologic morbidity. J Stroke Cerebrovasc Dis 2002;11:9-14

2. Linskey ME, Sekhar LN, Hirsch WL Jr, et al. Aneurysms of the intracavernous carotid artery: natural history and indications for treatment. Neurosurgery 1990;26:933-37

3. Nutik SL. Subclinoid aneurysms. J Neurosurg 2003;98:731-36

4. Beretta F, Sepahi AN, Zuccarello M, et al. Radiographic imaging of the distal dural ring for determining the intradural or extradural location of aneurysms. Skull Base 2005;15:253-61

5. Gonzalez LF, Walker MT, Zabramski JM, et al. Distinction between paraclinoid and cavernous sinus aneurysms with computed tomographic angiography. Neurosurgery 2003;52:1131-39

6. Hashimoto K, Nozaki K, Hashimoto N. Optic strut as a radiographic landmark in evaluating neck location of a paraclinoid aneurysm. Neurosurgery 2006;59:880-95

7. Oikawa S, Kyoshima K, Kobayashi S. Surgical anatomy of the juxta-dural ring area. J Neurosurg 1998;89:250-54

8. Thines L, Gauvrit JY, Leclerc X, et al. Usefulness of MR imaging for the assessment of nonophthalmic paraclinoid aneurysms. AJNR Am J Neuroradiol 2008;29:125-29

9. Hitotsumatsu T, Natori Y, Matsushima T, et al. Micro-anatomical study of the carotid cave. Acta Neurochir (Wien) 1997;139:869-74 